\title{
Modelación Fenomenológica de Cromatografía de Interacción Hidrofóbica
}

\author{
Andrea V. Mahn \\ Universidad de Santiago de Chile, Departamento de Ingeniería Química, Av. Libertador \\ Bernardo O’Higgins 3363, Estación Central, Casilla 10233, Santiago-Chile \\ (e-mail: amahn@lauca.usach.cl)
}

\section{Resumen}

El objetivo de este trabajo fue obtener un modelo fenomenológico que describa el perfil de elusión, en cromatografía de interacción hidrofóbica de una proteína inyectada como pulso. Se adaptó el modelo de platos, el que fue resuelto numéricamente, obteniéndose un buen ajuste a los datos experimentales. El modelo de platos permitió realizar simulaciones del proceso bajo distintas condiciones de operación, como velocidad de flujo, gradiente de elución y volumen de muestra inyectada. Se concluye que es posible aplicar la teoría de platos para describir perfiles de elución de proteínas en cromatografía de interacción hidrofóbica. El modelo obtenido podría ser utilizado para optimizar la separación de una mezcla de proteínas.

\section{Phenomenological Modelling of Hydrophobic Interaction Chromatography}

\begin{abstract}
The aim of this work was to obtain a phenomenological model to describe the elution profile in hydrophobic interaction chromatography of a protein injected as a pulse. The plate model, which was solved numerically, was used, and good fit to experimental data was found. The plate model allowed simulating the chromatographic process under different operating conditions, such as flow rate, elution gradient and sample volume. In is concluded that it is possible to apply the plate theory to describe elution profiles of proteins in hydrophobic interaction chromatography. The proposed model could be used to optimize the separation of protein mixtures.
\end{abstract}

Keywords: chromatography, hydrophobic interaction, protein purification, differential separation 


\section{INTRODUCCIÓN}

La cromatografía de interacción hidrofóbica (HIC) es una de las principales técnicas utilizadas para la separación y purificación de proteínas en procesos biotecnológicos. En HIC, las proteínas se unen reversiblemente a ligandos hidrofóbicos que se encuentran inmovilizados en el soporte cromatográfico, debido a la presencia de una elevada concentración de sal. La elución se logra disminuyendo la fuerza iónica en la fase móvil, formando un gradiente decreciente (Fausnaugh y Regnier, 1986).

Debido a la relevancia biotecnológica de HIC, se han hecho esfuerzos para determinar de qué forma las características de las proteínas y las condiciones de operación del sistema afectan el perfil de elución y el tiempo de retención de las proteínas (Hjerten et al., 1986; Melander et al., 1989; Mahn et al., 2005; Xia et al., 2005). Se ha logrado establecer que las principales variables de proceso son el tipo y la concentración máxima de sal utilizada para formar el gradiente de elución (Melander et al., 1989; Fausnaugh y Regnier, 1986) y el tipo de matriz cromatográfica (Machold et al., 2002), mientras que de las propiedades de las proteínas, la más importante sería la hidrofobicidad (Queiroz et al., 2001; Lienqueo et al., 2002).

Se han desarrollado diferentes modelos para describir y predecir el tiempo de retención de proteínas en HIC, basados tanto en las propiedades de las proteínas como también en las características del sistema cromatográfico. Algunos de estos modelos se describen a continuación.

\section{Modelos basados en la hidrofobicidad de las proteínas}

Chen et al. (2008), propusieron modelos de tipo QSPR (relación de propiedades estructurales cuantitativas) para predecir el tiempo de retención de proteínas en HIC, tanto en elución isocrática como en gradiente. Este modelo se basa en descriptores moleculares que reflejan grupos de propiedades fisicoquímicas y estructurales de las proteínas, entre las cuales se encuentra la hidrofobicidad.

Por otra parte, se han desarrollado modelos empíricos de la forma mostrada en la ecuación (1), donde $\Phi_{\text {surface }}$ corresponde a la hidrofobicidad superficial promedio de la proteína, DRT es el tiempo de retención adimensional, que corresponde al máximo del pico de elución normalizado por la duración del gradiente, y A, B y C son los parámetros del modelo (Lienqueo et al., 2002).

$\mathrm{DRT}=\mathrm{A}+\mathrm{B} \Phi_{\text {surface }}+\mathrm{C} \Phi_{\text {surface }^{2}}$

(1)

Los parámetros de este modelo no tienen significado físico, y dependen de las condiciones de operación del sistema. Por lo tanto, se trata de un modelo empírico que sólo puede ser utilizado si se cumplen las siguientes condiciones: (1) las corridas cromatográficas se realizan en las mismas condiciones de operación empleadas en la generación del modelo; y (2) se conoce la estructura tridimensional de la proteína de interés, lo que permite determinar su $\Phi_{\text {surface. }}$ Si bien este modelo ha sido utilizado con éxito en el diseño racional de procesos biotecnológicos (Lienqueo et al., 2003), su aplicación se ve limitada debido a su restringida generalidad.

\section{Modelos basados en las condiciones de operación del sistema}

Xiao et al. (2007), desarrollaron un modelo termodinámico biconformacional, que considera el efecto de la temperatura y de la concentración de sal sobre el tiempo de retención de proteínas en HIC. Este modelo describe el cambio en la energía libre de Gibbs en términos de la concentración de sal y de la temperatura, mediante ecuaciones lineales cuyos parámetros se determinan de manera empírica. Este modelo no permite la estimación directa del tiempo de retención de las proteínas. To et al. (2007), propusieron un modelo que describe el coeficiente de retención isocrático de proteínas en HIC en función de la concentración de sulfato de amonio. Los parámetros del modelo dependen de las características del adsorbente, y por lo tanto el modelo carece de generalidad. Además, no resulta aplicable a procesos de HIC con elución en gradiente, que son los más utilizados. 
Mahn et al. (2007), desarrollaron un modelo que considera las variables de operación estadísticamente significativas para el proceso de HIC. Este modelo tiene la ventaja de no requerir información estructural de las proteínas, pero de igual forma se trata de un modelo empírico cuyos parámetros no tienen significado físico. Este modelo tiene la forma mostrada en la ecuación (2):

$\mathrm{DRT}=\mathrm{A}+\mathrm{B} \mathrm{I}_{\mathrm{s}}+\mathrm{C} \sigma_{\mathrm{s}}+\mathrm{D} \mathrm{L}_{\mathrm{d}}$

Donde $I_{s}$ es la fuerza iónica de la fase móvil, $\sigma_{s}$ es la tensión superficial ejercida por la sal en solución, $L_{d}$ es la densidad superficial de los ligandos hidrofóbicos en la matriz cromatográfica, $A, B$, C y $\mathrm{D}$ son los parámetros del modelo. Este modelo se ha utilizado para describir la retención diferencial de mezclas de proteínas en HIC, y para determinar las condiciones de operación óptimas que permiten la mayor separación entre ellas. Si bien este modelo permite la optimización del proceso, sólo resulta aplicable dentro del rango de variables de proceso estudiadas.

Los tres modelos descritos anteriormente son de tipo empírico, por lo cual carecen de generalidad, y su aplicación a nuevos sistemas se ve restringida. Además, tanto los modelos basados en las condiciones de operación como aquellos basados en la hidrofobicidad de las proteínas no son capaces de entregar un perfil de elución, sino entregan una estimación del tiempo de retención. Con ello se dificulta el cálculo de la resolución y de la pureza, parámetros fundamentales para el diseño y optimización de procesos cromatográficos. Frente a esto se propone que un modelo fenomenológico podría ser de mayor utilidad. Este tipo de modelos se han utilizado para describir los perfiles de elución en distintos tipos de cromatografía, principalmente cromatografía de intercambio iónico (Shene et al., 2006).

Jakobsson et al. (2005), propusieron un método híbrido para analizar y optimizar HIC, que consta de dos etapas. La primera etapa consiste en aplicar un diseño estadístico de experimentos para determinar las condiciones de operación óptimas para un determinado proceso HIC (se consideran tres factores, lo que da un total de 16 corridas experimentales). La segunda etapa consiste en resolver la ecuación de continuidad, que considera los fenómenos de dispersión y convección de la fase móvil, y la adsorción (como reacción química), y calibrarla con la información obtenida en la primera etapa. Si bien los autores lograron simular exitosamente los perfiles de elución de dos proteínas, el método presenta la desventaja de requerir un elevado número de determinaciones experimentales en la primera etapa.

En este trabajo se propone que los perfiles de elución de proteínas en HIC pueden ser simulados y optimizados mediante un modelo basado en la teoría de platos. Este es un modelo clásico utilizado en operaciones unitarias (Astorga et al., 2006).

\section{Modelo de platos teóricos}

Se adaptó el modelo de platos teóricos al proceso HIC. El modelo fue resuelto numéricamente, utilizando datos experimentales para el ajuste de parámetros. El sistema a modelar consistió en una columna cromatográfica de nivel laboratorio (1 $\mathrm{mL}$ de volumen) empacada con resina para interacción hidrofóbica. El proceso consistió en la inyección de muestra (compuesta por buffer, sal y una proteína) en forma de pulso, bajo condiciones de elevada fuerza iónica, para su posterior elución mediante disminución gradual de la fuerza iónica de la fase móvil.

El modelo de platos asume que la columna cromatográfica está compuesta por un número de platos teóricos ( $\mathrm{Np}$ ) en equilibrio, cada uno de los cuales tiene la misma razón entre la fase móvil y la estacionaria. La fase móvil fluye desde un plato al siguiente, mientras que la fase estacionaria permanece fija en el respectivo plato (Tejeda et al., 1995). El balance de masa en el n-ésimo plato puede ser representado por la ecuación (3):

$$
F\left[y_{n-1}-y_{n}\right]=\frac{V_{0}}{N_{p}}\left(\frac{d y_{n}}{d t}\right)+\frac{V_{t}-V_{0}}{N_{p}}\left(\frac{d q_{n}}{d t}\right)
$$


Donde $\left(\mathrm{Vt}-\mathrm{V}_{0}\right)$ es la porosidad del lecho, $\mathrm{F}$ es el flujo, $\mathrm{y}_{\mathrm{n}}$ es la concentración de proteína en el nésimo plato; $q_{n}$ es la concentración de proteína en la fase estacionaria en el $n$-ésimo plato. Se consideraron los siguientes supuestos:

1) Cada plato se encuentra en equilibrio, determinado por una isoterma lineal.

2) La razón de volúmenes entre las fases permanece constante.

3) El flujo es constante y no existe mezclado entre los platos.

4) El equilibrio de la proteína entre las dos fases es instantáneo.

5) El coeficiente de distribución de la sal no es afectado por la presencia de proteína.

6) El número de platos teóricos para la proteína y para sal son constantes.

En equilibrio, la concentración de proteína en la fase estacionaria se relaciona con aquella en la fase móvil a través del coeficiente de distribución $\mathrm{K}$, que a su vez depende de la fuerza iónica y de la concentración de proteína:

$\mathrm{q}_{\mathrm{n}}=\mathrm{K}\left(\mathrm{y}_{\mathrm{n}}, \mathrm{l}\right)^{*} \mathrm{y}_{\mathrm{n}}$

Combinando las ecuaciones (3) y (4) se tiene:

$\frac{d y_{n}}{d t}=\frac{N_{p}\left[y_{n-1}-y_{n}\right]-y_{n} H\left(\frac{d K\left(y_{n}, I\right)}{d I} * \frac{d I}{d \Theta}\right)}{1+H\left[K\left(y_{n}, I\right)+y_{n} \frac{d K\left(y_{n}, I\right)}{d y_{n}}\right]}$

El balance de materia para la sal está dado por la ecuación (6):

$$
\frac{d I_{n}}{d \Theta}=R^{\prime *}\left(I_{n-1}-I_{n}\right)
$$

Las ecuaciones (5) y (6) forman un sistema de ecuaciones no lineales, que fue resuelto utilizando el método de Runge - Kutta. Las condiciones iniciales fueron:

$\mathrm{y}_{\mathrm{n}=1}=\mathrm{y}_{\mathrm{n}=2}=\cdots=\mathrm{y}_{\mathrm{np}}=0 ; \quad$ si $\theta=-\mathrm{Xo}$

Las condiciones de borde fueron:

$\begin{aligned} \mathrm{y}_{\mathrm{n}=0}=\mathrm{y}_{0} & \text { si } & -\mathrm{Xo}_{0}<\theta \leq 0 \\ \mathrm{y}_{\mathrm{n}=0}=0 & \text { si } & \theta>0\end{aligned}$

El número de platos teóricos se determinó mediante la ecuación (7):

$$
N_{p}=\frac{z}{\frac{2 D l}{u}+\frac{d_{p}^{2} H K_{\operatorname{Imax}}{ }^{2} u}{\left[30 D_{\text {crit }} K_{\text {crit }}\left(1+H K_{\operatorname{Imax}}\right)^{2}\right]}}
$$

Las ecuaciones (5) - (7) fueron implementadas en Matlab ${ }^{\mathrm{TM}}$ y resueltas numéricamente mediante el método de Runge - Kutta.

El objetivo de este trabajo fue obtener un modelo fenomenológico basado en la teoría de platos, que describa el perfil de elución en HIC de una proteína inyectada como pulso. Se adaptó el modelo de platos a HIC, para permitir la simulación de procesos de purificación de proteínas basados en esta técnica. 


\section{METODOLOGÍA}

Se utilizó la resina Phenyl Sepharose 6 FF (GE Healthcare, Uppsala, Sweden); sulfato de amonio, lisozima, albúmina de suero bovino y bis-tris Sigma (St. Louis, MO), además de agua mili-pura. Para el estudio de la cinética de adsorción en discontinuo, se utilizaron soluciones de lisozima de concentración $2 ; 1.6 ; 1.2 ; 0.8 ; 0.4$ y $0.0 \mathrm{mg} / \mathrm{mL}$ (30 mL volumen final) y se les agregó $1 \mathrm{~mL}$ de resina a cada solución bajo agitación constante. Se tomaron muestras de $1 \mathrm{~mL}$ a diferentes tiempos de incubación: 30; 65; 95; 190 y 340 minutos. Las muestras fueron centrifugadas y se determinó la concentración de lisozima en el sobrenadante utilizando el método Bradford, usando BSA como estándar. En las corridas cromatográficas se utilizó el sistema para cromatografía líquida FPLC (GE Healthcare, Uppsala, Sweden) con un bucle de inyección de $100 \mu \mathrm{L}$. Se utilizó una columna de $1 \mathrm{~mL}$ para empacar la resina. Los experimentos se llevaron a cabo a temperatura ambiente $\left(20^{\circ} \mathrm{C}\right)$ utilizando una velocidad de flujo igual a $0.75 \mathrm{~mL} / \mathrm{min}$ y un gradiente decreciente de sal en 10 volúmenes de columna. La solución de salida fue monitoreada a $280 \mathrm{~nm}$ y se registró el volumen de retención y la conductividad. Se inyectó una solución de lisozima $0.5 \mathrm{mg} / \mathrm{mL}$. La fase móvil final fue buffer bis-tris $20 \mathrm{mM} \mathrm{pH} \mathrm{7.0,} \mathrm{y} \mathrm{la} \mathrm{fase} \mathrm{móvil} \mathrm{inicial} \mathrm{fue} \mathrm{el} \mathrm{mismo} \mathrm{buffer} \mathrm{adicionado} \mathrm{de} 2 \mathrm{M}$ sulfato de amonio. Todas las determinaciones experimentales se hicieron en triplicado.

\section{RESULTADOS Y DISCUSIÓN}

El coeficiente de partición $\mathrm{K}$ fue determinado a partir de los datos de adsorción discontinua, utilizando regresión lineal $\left(r^{2}=0.998\right)$. La isoterma de adsorción se muestra en la ecuación $(8)$, donde $\mathrm{K}$ es igual a $336 \mathrm{~mL}$ solución / mL resina. Las características del sistema cromatográfico se muestran en las tablas 1 y 2 .

$q_{i}=336 * P_{i}$

Tabla 1: Parámetros fisicoquímicos

\begin{tabular}{|c|c|}
\hline Parámetro & Valor determinado \\
\hline $\mathrm{D}_{\mathrm{AB}}$ & $3.82{ }^{\star} 10^{-7}\left[\mathrm{~m}^{2} / \mathrm{h}\right]$ \\
\hline $\mathrm{u}$ & $2.3[\mathrm{~m} / \mathrm{h}]$ \\
\hline $\mathrm{k}$ & $23[1 / \mathrm{h}]$ \\
\hline $\mathrm{k}_{\mathrm{m}}$ & $0.039[\mathrm{~m} / \mathrm{h}]$ \\
\hline
\end{tabular}

Tabla 2: Propiedades fisicoquímicas del sistema cromatográfico

\begin{tabular}{|c|c|}
\hline Diámetro de partícula & $93^{\star} 10-6[\mathrm{~m}]$ \\
\hline Diámetro interno columna & $5^{\star} 10-3[\mathrm{~m}]$ \\
\hline Largo columna & $5^{\star} 10-2[\mathrm{~m}]$ \\
\hline Porosidad del lecho & $0.3[-]$ \\
\hline Densidad matriz & $1130[\mathrm{Kg} / \mathrm{m} 3]$ \\
\hline Temperatura de operación & $293 \mathrm{~K} ; 20^{\circ} \mathrm{C}$ \\
\hline Concentración de la muestra & $0.5[\mathrm{mg} / \mathrm{mL}]$ \\
\hline Flujo volumétrico & $4.5^{\star} 10-5[\mathrm{~m} 3 / \mathrm{h}]$ \\
\hline Flujo lineal & $2.3[\mathrm{~m} / \mathrm{h}]$ \\
\hline Viscosidad solvente & $1.0828 * 10-3\left[\mathrm{~Pa}{ }^{\star} \mathrm{s}\right]$ \\
\hline Peso molecular lisozima & $14000[\mathrm{Da}]$ \\
\hline Área específica resina $(\mathrm{s})$ & $40\left[\mathrm{~m}^{2} / \mathrm{Kg}\right.$ resina] \\
\hline
\end{tabular}

El perfil de elución de lisozima se simuló utilizando los parámetros determinados experimentalmente (tabla 1). Para obtener un buen ajuste, se optimizó el número de platos (Np). Los resultados se muestran en la figura 1. La máxima concentración a la salida de la columna fue muy cercana a la obtenida experimentalmente para un $\mathrm{Np}$ igual a 15. Sin embargo, para este valor el pico simulado resultó ser más angosto que el experimental, probablemente debido a la relativamente elevada concentración de proteína inyectada. Para valores de Np menores se observó una deformación en la 
forma del pico, probablemente debido a que con valores de $\mathrm{Np}$ bajos se obtiene una curva de tipo Poisson, y la teoría de platos es válida para curvas de tipo Gauss. Se observó un desplazamiento del pico hacia el origen, mientras que en el otro extremo la curva simulada mostró una forma suavizada. El parámetro $\mathrm{Np}$ tendría relación con los fenómenos de transferencia de materia y de dispersión, y por lo tanto entrega una idea de la resistencia a la transferencia de materia del sistema. Este parámetro se vio afectado por la velocidad de flujo y también por la difusividad crítica.

La curva de elución de una muestra de proteína inyectada en forma de pulso en una columna HIC puede ser descrita por la ecuación (9), que corresponde a una solución analítica del modelo. En ella, t es el tiempo de operación de la columna, $t_{0}$ es el tiempo de elución de la proteína e $y_{0}$ es la máxima concentración de proteína a la salida de la columna. En esta ecuación, $\mathrm{N}$ es la incógnita, la que es igual al inverso de la desviación estándar. Al ajustar la solución analítica a los datos experimentales, se obtuvo un $\mathrm{N}$ igual a 27, lo que corresponde a una desviación estándar de 0.192.

$y=y_{0} e^{\left[-\frac{\left(t / t_{0}-1\right)^{2}}{2 / N}\right]}$

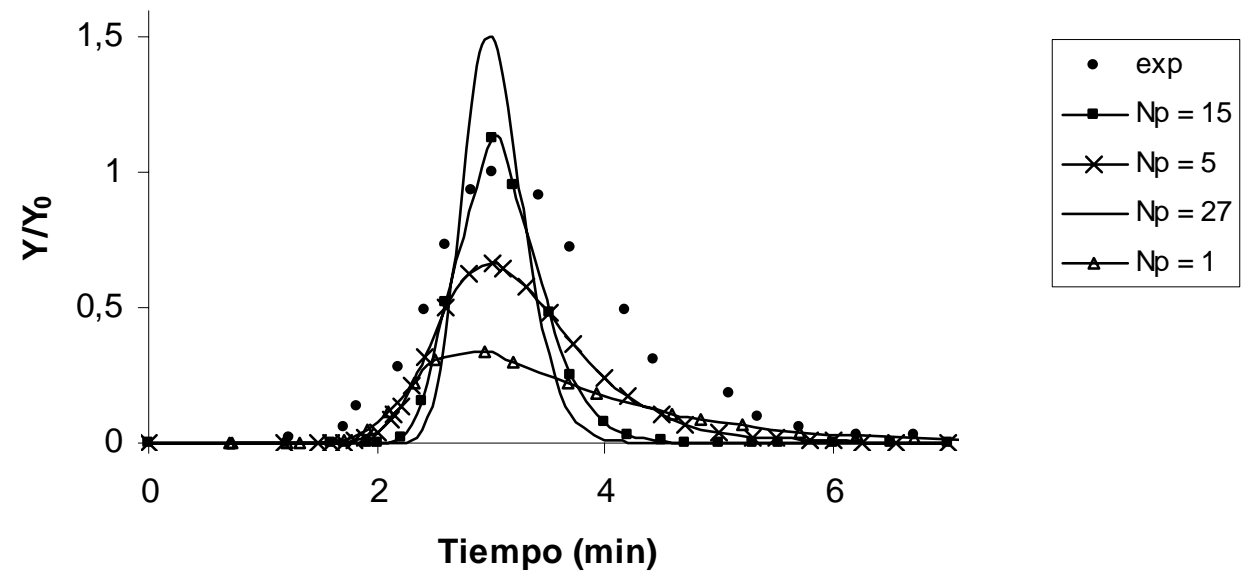

Fig. 1: Solución numérica del modelo. La línea discontinua corresponde a los datos experimentales obtenidos para lisozima, y las líneas continuas corresponden al modelo ajustado.

El mejor valor de $\mathrm{Np}$ entregado por la solución numérica del modelo de platos fue 15, que corresponde a una desviación estándar de 0.258, algo superior obtenida con la solución analítica. Una menor desviación estándar se relaciona con un pico más angosto, y por lo tanto con un menor efecto de dispersión. De esta forma, la solución numérica representaría de manera más adecuada el comportamiento experimental.

El modelo de platos se ha utilizado para la modelación de cromatografía de intercambio iónico (IEC) (Shene et al., 2006), sin embargo hasta ahora no existen antecedentes sobre la aplicación de este modelo a HIC. Los autores obtuvieron un valor de $\mathrm{Np}$ igual a 8 para una proteína similar a lisozima (lactoglobulina), valor que resulta inferior al obtenido en este trabajo $(\mathrm{Np}=15)$ para lisozima. Esta situación puede ser atribuida a que Shene et al. (2006), utilizaron una menor concentración de proteína en la muestra inyectada. Además, existen diferencias en las características físicas y químicas de los sistemas cromatográficos utilizados (IEC y HIC). Las demás condiciones de operación (excepto la concentración de proteína en la muestra) utilizadas por Shene et al. (2006), tales como temperatura, volumen de muestra inyectada, velocidad de flujo y gradiente, fueron similares a las utilizadas en este trabajo.

Se investigó el efecto del volumen de muestra inyectada, velocidad de flujo y gradiente de elución sobre el perfil de elución de una proteína. Los resultados se presentan en la figura 2. 
La figura 2(a) muestra que la velocidad de flujo afecta el tiempo de retención de manera inversamente proporcional: a menor flujo, mayor tiempo de retención, manteniendo las demás condiciones de operación constantes. En la práctica, una velocidad de flujo menor favorece los fenómenos de transporte de materia; ya que se incrementa el tiempo de residencia de la proteína dentro de la columna, y por lo tanto las moléculas alcanzan a recorrer los intersticios del lecho y entrar en contado con los ligandos hidrofóbicos de la matriz. Cuando se utilizan flujos elevados se produce una adsorción incompleta, situación que justifica el hecho que con un flujo igual a 0.75 $\mathrm{mL} / \mathrm{min} \mathrm{Y} / \mathrm{Yo}$ sea levemente mayor que para flujos más altos.
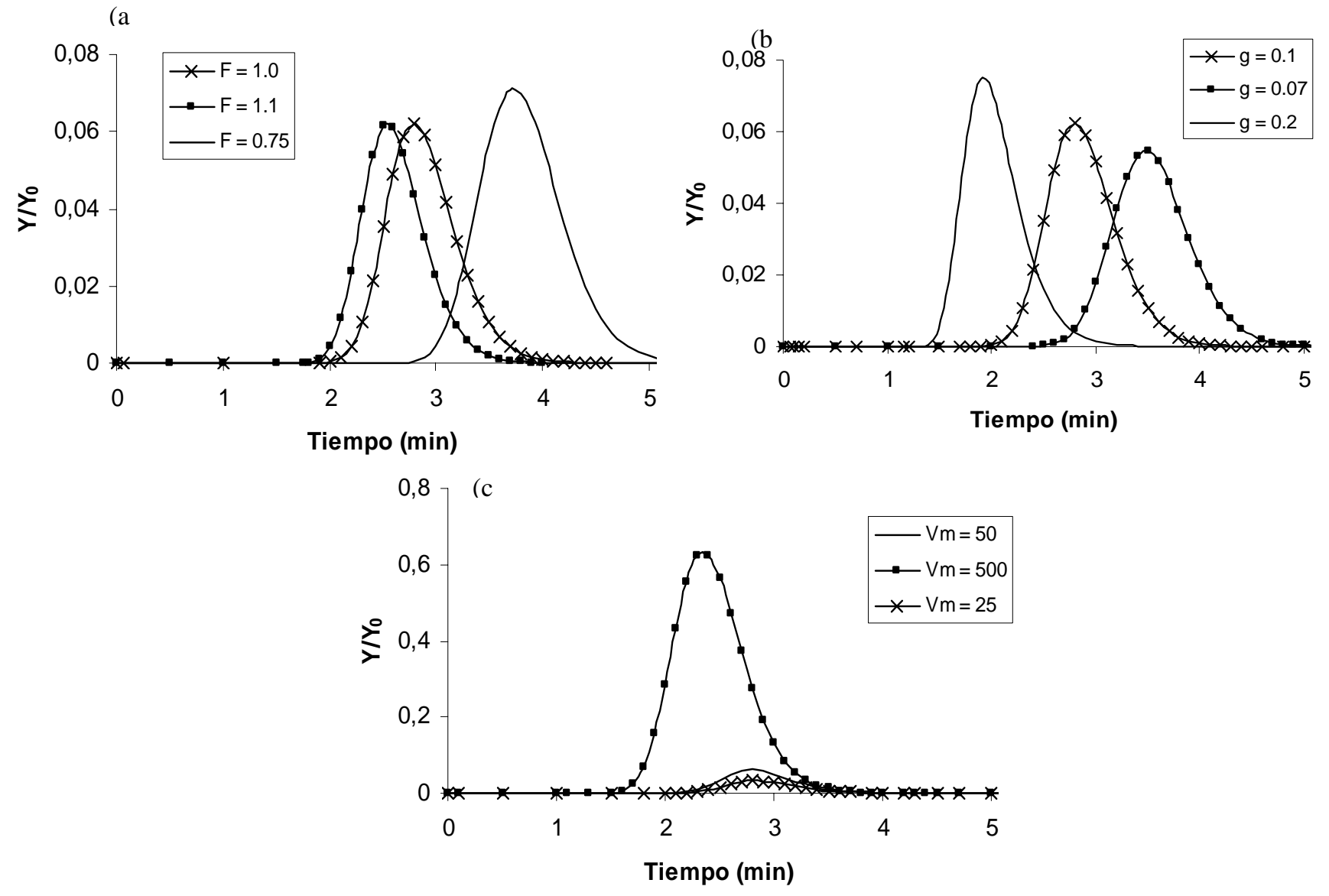

Fig. 2: Efecto de la velocidad de flujo [mL/min] (a), gradiente de elución [-M/mL] (b) y volumen de muestra inyectada $[\mathrm{mL}](\mathrm{c})$ sobre el perfil cromatográfico.

La figura 2 (b) muestra que el gradiente de elución afecta el tiempo de retención, la máxima concentración a la salida y el ancho del pico. Un mayor gradiente implica que el tiempo en el cual la proteína se encuentra bajo condiciones de elución es menor, por lo cual el perfil tiende a asemejarse más a una respuesta tipo pulso, es decir, un pico más angosto, lo que a su vez significa que existe un menor efecto de dispersión. Como se simuló igual cantidad de proteína inyectada en los tres casos analizados, resulta lógico pensar que a menor dispersión se obtiene una mayor concentración máxima de salida (Y/Yo), lo que se ve bien representado en la figura 2 (b).

La figura 2 (c) muestra que el volumen de muestra inyectado afecta el ancho del pico, la máxima concentración a la salida, y el tiempo de retención de la proteína. Un mayor volumen de muestra se traduce en un pico más ancho (mayor dispersión) y más alto (mayor Y/Yo). Además, la disminución del volumen de muestra implica un retraso en la elución de la proteína, esto es, un tiempo de retención mayor. Esta situación puede atribuirse a una posible saturación de la columna debido a la elevada cantidad de proteína inyectada, lo que adelantaría su elución.

En cuanto al número de platos (Np), es posible afirmar que ni el volumen de muestra ni la pendiente del gradiente influyen sobre este parámetro, a diferencia de la velocidad de flujo y la difusividad crítica, que tienen un efecto importante sobre Np. En la Tabla 3 se muestra dicho efecto. 
El parámetro Dcrit, que representa la difusividad crítica en la fase estacionaria, influye directamente sobre el número de platos (Np). Un menor Dcrit implica un mayor número de platos, lo que a su vez se traduce en un pico más angosto. Esto significa que un aumento en la difusividad crítica en la fase estacionaria implica una disminución de la resistencia a la transferencia de masa interna, luego la curva de elución se acerca más a una respuesta tipo pulso. Los resultados obtenidos para Dcrit concuerdan con lo reportado por Shene et al., (2006), quienes obtuvieron valores experimentales del orden de $2 \times 10^{-7}\left[\mathrm{~cm}^{2} / \mathrm{min}\right]$ en cromatografía de intercambio iónico $(\mathrm{Np}=8)$, para la proteína lactoglobulina, que presenta similares características fisicoquímicas que lisozima.

Tabla 3: Efecto de F y Dcrit sobre el número de platos teóricos

\begin{tabular}{|c|c|}
\hline $\mathrm{F}[\mathrm{mL} / \mathrm{min}]$ & $\mathrm{Np}$ \\
\hline 0.6 & 11 \\
\hline 0.75 & 9 \\
\hline 1.0 & 7 \\
\hline 1.1 & 7 \\
\hline Dcrit $\left[\mathrm{cm}^{2} / \mathrm{s}\right]$ & $\mathrm{Np}$ \\
\hline $1 * 10^{-5}$ & 160 \\
\hline $3 * 10^{-7}$ & 9 \\
\hline $1 * 10^{-8}$ & 1 \\
\hline
\end{tabular}

\section{CONCLUSIONES}

La retención de proteínas en HIC puede ser modelada a partir de la hidrofobicidad de las proteínas, y también a partir de las características del sistema, a través de modelos empíricos. Sin embargo, la aplicabilidad de este tipo de modelos es limitada, dado que no siempre se puede contar con la información necesaria y además debido a que ellos carecen de generalidad. Se propuso que un modelo fenomenológico podría ser de utilidad en la simulación y optimización de HIC.

Fue posible adaptar el modelo de platos teóricos para simular el perfil de elución de una proteína en HIC. Las simulaciones permitieron concluir que la velocidad de flujo afecta de manera indirecta el tiempo de retención de una proteína. El gradiente de elución y el volumen de muestra inyectado afectan al tiempo de retención, la concentración máxima de salida y el ancho del pico. Un mayor gradiente se traduce en un menor efecto de dispersión, mientras que un mayor volumen implica una mayor dispersión. Además, la disminución del volumen de muestra se traduce en un mayor tiempo de retención.

Se obtuvo un buen ajuste entre los datos experimentales y las simulaciones de los perfiles de elución, lo que sugiere que es posible usar la teoría de platos para describir perfiles de elución de proteínas en HIC. El modelo de platos refleja de manera indirecta los fenómenos de transferencia de masa, dispersión y adsorción (reacción química), a través del parámetro $\mathrm{Np}$, el cual depende de dichos factores. Si bien los ajustes obtenidos fueron satisfactorios, estos resultados podrían mejorarse aumentando las determinaciones experimentales de los parámetros del modelo, como Dcrit y Kcrit, de tal forma que la única incógnita sea Np, facilitando el ajuste.

\section{AGRADECIMIENTOS}

Este trabajo fue financiado por el proyecto Fondecyt 1061154.

\section{NOMENCLATURA}

$\begin{array}{ll}a_{v} & \text { área específica volumétrica } \\ D_{A B} & \text { difusividad } \\ D_{\text {crit }} & \text { difusividad crítica } \\ d p & \text { siámetro de partícula }\end{array}$


F flujo volumétrico

g pendiente del gradiente de elución

l fuerza iónica

$\mathrm{K} \quad$ coeficiente de partición

K' coeficiente de partición de la sal

$\mathrm{K}_{\text {crit }} \quad$ coeficiente de partición crítico

$\mathrm{K}_{\operatorname{lmax}} \quad$ coeficiente de partición a fuerza iónica máxima

$\mathrm{N} \quad$ número de platos teóricos (solución analítica)

$\mathrm{N}_{\mathrm{p}} \quad$ número de platos teóricos (solución numérica)

$\mathrm{N}_{\mathrm{p}}$ ' número de platos para la sal

$q_{i} \quad$ concentración de proteína adsorbida a la matriz

$\mathrm{q}_{\mathrm{n}} \quad$ concentración de proteína adsorbida en el n-ésimo plato

$v_{c} \quad$ volumen de la columna

$\mathrm{V}_{0} \quad$ volumen de retención

$\sigma \quad$ desviación estándar

$\mathrm{t} \quad$ tiempo

$\Theta \quad$ tiempo adimensional (solución numérica)

u velocidad de flujo lineal

$\mu \quad$ viscosidad

$V_{E} \quad$ volumen del plato

$V_{m} \quad$ volumen de muestra inyectada

yo máxima concentración de proteína a la salida de la columna

$\mathrm{y}_{\mathrm{n}} \quad$ concentración de proteína en la fase móvil en el n-ésimo plato

$\mathrm{X}_{0} \quad$ razón $\mathrm{V}_{\mathrm{m}} / \mathrm{V}_{0}$

z $\quad$ posición a lo largo de la columna

\section{REFERENCIAS}

Astorga, C. M, E. Quintero-Marmol, L. G Vela y L. Torres; Observador No Lineal para la Estimación de Concentraciones en un Proceso de Destilación Metanol/Etanol. Inf. Tecnol.: 17(6), 35-41 (2006).

Chen J, T. Yang y S.M. Cramer; Prediction of protein retention time in gradient hydrophobic interacting systems. J. Chromatogr. A: 1177, 207-214 (2008).

Fausnaugh J.L. y F.E. Regnier; Solute and mobile phase contributions to retention in hydrophobic interaction chromatography. J. Chromatogr.: 359, 131-146 (1986).

Hjerten S., K. Yao, O. Eriksson y B. Johansson; Gradient and isocratic high - performance hydrophobic interaction chromatography of proteins on agarose columns. J. Chromatogr.: 359, 99109 (1986).

Jakobsson N., M. Degerman y B. Nilsson; Optimisation and robustness analysis of a hydrophobic interaction chromatography step. J. Chromatorg. A: 1099, 157-166 (2005).

Lienqueo M.E., A. Mahn y J.A. Asenjo; Mathematical Correlations for Predicting Protein Retention Time in Hydrophobic Interaction Chromatography. J. Chromatogr. A: 978, 71-79 (2002).

Lienqueo M.E., A .Mahn, L. Vásquez y J.A. Asenjo; Methodology for predicting the separation of proteins by hydrophobic interaction chromatography and its application to a cell extract. J. Chromatogr. A: 1009, 189-196 (2003).

Machold C., K. Deinhofer, R. Hahn y A. Jungbauer; Hydrophobic interaction chromatography of proteins I: Comparison of selectivity. J. Chromatogr. A: 972, 3-19 (2002).

Mahn A., M. E. Lienqueo y J. A. Asenjo; Optimal operation conditions for protein separation in hydrophobic interaction chromatography. J Chromatogr. B: 849, 236-242 (2007). 
Mahn A., G. Zapata y J.A. Asenjo; A Theory of Protein - Resin Interaction in Hydrophobic Interaction Chromatography. J. Chromatogr. A: 1066, 81-88 (2005).

Melander W., Z. EI Rassi y CS. Horvath; Interplay of hydrophobic and electrostatic interactions in biopolymer chromatography: Effect of salts on the retention of proteins. J. Chromatogr.: 469, 327(1989).

Queiroz J.A., C.T. Tomaz y J.M.S. Cabral; Hydrophobic interaction chromatography of proteins. J. Biotechnol.: 87, 143-159 (2001).

Shene C, A. Lucero, B.A. Andrews y J.A. Asenjo; Mathematical modeling of elution curves for a protein mixture in ion exchange chromatography and for the optimal selection of operational conditions. Biotechnol. Bioeng.: 95, 704-713 (2006).

Tejeda A., R.M. Montesinos y R. Guzmán; Bioseparaciones. Unison, México, 419 páginas (1995).

To, B.C.S. y A.M. Lenhoff; Hydrophobic interaction chromatography of proteins I. The effects of protein and adsorbent properties on retention and recovery. J. Chromatogr. A: 1141, 191-205 (2007).

Xia F., D. Nagrath y S.M. Cramer; Effect of Ph changes on water release values in hydrophobic interaction chromatography systems. J. Chromatogr. A: 1079, 229-235 (2005).

Xiao Y., A. Rathore, J.P. O'Conell y E.J. Fernández; Generalizing a two-conformation model for describing salt and temperature effects on protein retention and stability in hydrophobic interaction chromatography. J. Chromatogr. A: 1157, 197-206 (2007). 